\title{
The Static WKB Solution to Catenary Problems with Large Sag and Bending Stiffness
}

\author{
Yuhung Hsu and Chanping Pan \\ Department of Construction Engineering, National Taiwan University of Science and Technology, Taipei City 10607, Taiwan \\ Correspondence should be addressed to Yuhung Hsu; jeromeehsu@gmail.com
}

Received 30 June 2014; Accepted 11 September 2014; Published 28 September 2014

Academic Editor: Bruno Briseghella

Copyright ( 2014 Y. Hsu and C. Pan. This is an open access article distributed under the Creative Commons Attribution License, which permits unrestricted use, distribution, and reproduction in any medium, provided the original work is properly cited.

\begin{abstract}
Large sag with a bending stiffness catenary is a subject that draws attention in the realm of fatigue analysis, estimation of suspension cable sag for bridge cable hoisting, and ocean engineering of the employment of mooring systems. However, the bending stiffness is the cause of boundary layers at the anchorage of cables, thereby finding a solution of the differential equation can be extremely difficult. Previous studies have tackled this problem with the perturbation method; yet, due to the complexity of the matching process and solution finding, the method might not be an ideal solution for engineering applications. Moreover, the finite difference method and the finite element method in numerical analysis can often be ineffective because of inappropriate parameter configuration and the drastic variation of functions in the boundary layers. Therefore, this study proposed a novel bending moment expression of a large sag catenary. The expression was derived from the sag identified using bending moment equations, and a solution was found by applying the WKB method (Wentzel-Kramers-Brillouin method) to overcome the complex problem of boundary layers. Consequently, a simple solution of various mechanical properties in a cable with bending stiffness and large sag could be obtained.
\end{abstract}

\section{Introduction}

Slim tension members have been comprehensibly applied to civil and ocean engineering to create cables of cable-stayed and suspension bridges that satisfy traffic demands and mooring systems that satisfy the demands of mining deepsea petrol and natural gas. Such structural problems could be understood as general cable problems where the mechanical behavior of cables varies significantly with the magnitude of tension. In the study of cables of bridges, researchers often regard the ratio of midpoint sag to span as an appropriate standard for simplifying the parabola theory. When the ratio of midpoint sag to span is $1 / 8$, the horizontal force happens to be the total cable weight. When the ratio of midpoint sag to span is smaller than $1 / 8$ (horizontal force greater than the total cable weight), the resulting parabola is appropriate and correct. However, when the ratio of midpoint sag to span is greater than 1/8 (horizontal force smaller than the total cable weight), a greater error can be found in the parabola [1]. The relatively high tension applied on the cables of a cable-stayed bridge can result in a deflected shape that can be simplified as a parabola because of its relatively small sag and their bending stiffness have only minor effects and are negligible; however, deflected shapes of cables with relatively low tension (main cables of a suspension bridge or marine risers in ocean engineering) can be regarded as a catenary because the large sag increases the influence of the bending stiffness and cannot be neglected. In addition, the bending moment and stress of the anchor points are important parameters in fatigue analysis. Damages to cables caused by fatigue result in significant losses; thus, the understanding of mechanical behavior in cables with bending stiffness demands immediate attention.

The problem, however, of incorporating the bending stiffness effect in cables results from the complex differential equations. Bending stiffness is the cause of boundary layers in anchorages and rapid variations of bending moments occur near the differential regions of the cable anchorage. Burgess [2] utilized the finite difference method to analyze cables with bending stiffness and low tension and indicated the importance of parameter configuration to avoid the loss of effectiveness when employing general finite difference 
or finite element methods. Reasonable precision should be obtained by adopting a smaller step size and finer grid. Moreover, an approximation algorithm (e.g., perturbation method) should be adopted to find the analytical expression, which cannot be found by an analytical approach because of the complexity of differential equations.

In dealing with the boundary layer problems, the perturbation method consists of two main techniques [3-5]: matched asymptotic expansions and multiple scales method. Most scholars adopted the matched asymptotic expansions to analyze boundary layers in cables with bending stiffness, as M. S. Triantafyllou and G. S. Triantafyllou [6] analyzed the hanging string, Wolfe [7] analyzed the inextensible cable, Irvine [8] analyzed the stay-cables, Stump and Fraser [9] analyzed the high-speed transport of thin-sheet materials, and Stump and van der Heijden [10] analyzed bending and twisting rods. In later research, Denoël and Detournay [11] employed multiple scales method. Furthermore, Denoël and Canor [12] embraced a new perturbation method, patching asymptotics, for analysis. Nonetheless, methods of perturbation are not convenient choices for engineering applications. All perturbation methods require the matching of solutions of cable segments outside the boundary layers and beam segments inside the boundary layers and the matching procedures as well as the solutions are extraordinarily complicated.

To correct the weaknesses of a conventional perturbation method, this study proposed a novel catenary bending moment equation. The core idea originated from the comparison between two catenary models, which consisted of anchorages with the same horizontal distance and vertical elevation. The self-weight and horizontal force between the two models were also identical. The only difference is that one model contains bending stiffness but the other does not. The bending moment solution of the model with a bending stiffness catenary can be expressed by that of the model without a bending stiffness catenary. By winding around the difficulty of a conventional perturbation method, which requires a fourth order differential equation for finding the solution, the proposed equation could directly identify cable sag with the help of bending moment equations.

To satisfy the demands of different cable forces and to verify the correctness of the proposed bending moment equation, two respective second order differential equations of parabola and catenary incorporating bending stiffness were established and discussed. The parabola was expressed by a small sag linear moment-curvature relation, and the solution of the parabola was used to verify the catenary result. The catenary was expressed by a large sag nonlinear momentcurvature relation; moreover, the WKB method (WentzelKramers-Brillouin method is named after physicists Wentzel, Kramers, and Brillouin, who all developed it in 1926.) in the perturbation method [3-5] was adopted to solve the sag differential equations. The solution could resolve the complexity of boundary layers and does not require the matching of cable segments and beam segments as does a general perturbation method. With only one equation, the proposed method could describe the drastic variations in functions inside and outside the boundary layer and find the sag and bending moment effortlessly. Furthermore, this study established the influence of bending stiffness on cables' static behavior and the standard where the parabola could replace the catenary.

\section{Static Theories of Catenaries and Parabolas without Bending Stiffness}

2.1. The Catenary Solution. Figure 1 illustrates a catenary problem that considers only tensile forces without the bending moment. Self-weight effects are given and set, and the catenary is located in the first quadrant. The equation of static equilibrium parallel to the $x$-axis is

$$
\frac{\partial}{\partial x}\left(T \frac{\partial x}{\partial s}\right)=\frac{\partial}{\partial x}(H)=0 .
$$

$T$ is the tension, $H$ is the horizontal tension, and $H$ is a constant. The equation of static equilibrium perpendicular to the $y$-axis is

$$
\frac{\partial}{\partial x}\left(T \frac{\partial y}{\partial s}\right)=\frac{\partial}{\partial x}\left(H \frac{\partial y}{\partial x}\right)=H \frac{\partial^{2} y}{\partial x^{2}}=m g \frac{\partial s}{\partial x} .
$$

$m$ is the per-unity-length mass of the cable, $g$ is acceleration of gravity, and $s$ is the length of the catenary curve. $L$ is the horizontal distance between the anchorages. $m g$ is uniformly distributed along the curved cable, and $\partial s / \partial x=$ $\sqrt{1+(d y / d x)^{2}}=\sqrt{1+y^{\prime 2}}$. Dimensionless parameters are introduced as follows: $\bar{x}=x / L, \bar{y}=y / L$, and $\mathrm{mgL} / H=$ $q$, where $q$ implies the effects of cable self-weight on the equation of static equilibrium. Equation (2) is modified and becomes

$$
\bar{y}^{\prime \prime}=\frac{m g L}{H} \sqrt{1+\bar{y}^{\prime 2}}=q \sqrt{1+\bar{y}^{\prime 2}} .
$$

$\bar{y}$ is the dimensionless sag of the catenary, where the prime denote differentiation with respect to $x$. It can be substituted into the boundary conditions of the hinges on the two ends $(\bar{y}(0)=1$ and $\bar{y}(1)=\tan \theta)$, and the solution of $(3)$ is the solution of the catenary. Consider

$$
\bar{y}=\frac{1}{q}\left\{\cosh \left(q \bar{x}+a-\frac{q}{2}\right)-\cosh \left(a-\frac{q}{2}\right)\right\} .
$$

Consider $a=\sinh ^{-1}(q \tan \theta /(2 \sinh (q / 2)))$. The two hinges are at the same elevation level $\left(\theta=0^{\circ}, a=0\right)$; (4) can be simplified as

$$
\bar{y}=\frac{1}{q}\left\{\cosh \left[q\left(\bar{x}-\frac{1}{2}\right)\right]-\cosh \left(\frac{q}{2}\right)\right\} .
$$

2.2. The Parabola Solution. As shown in Figure 2, given that the self-weight distributes uniformly along the chordwise direction, $\partial s / \partial x=\sec \theta$ is a constant, and $\theta$ is the chordwise inclination of the suspension cable. The suspension cable length indicates the shortest distance between the two anchorages at the two ends. Equation (3) is modified as

$$
\bar{y}^{\prime \prime}=q \sec \theta \text {. }
$$




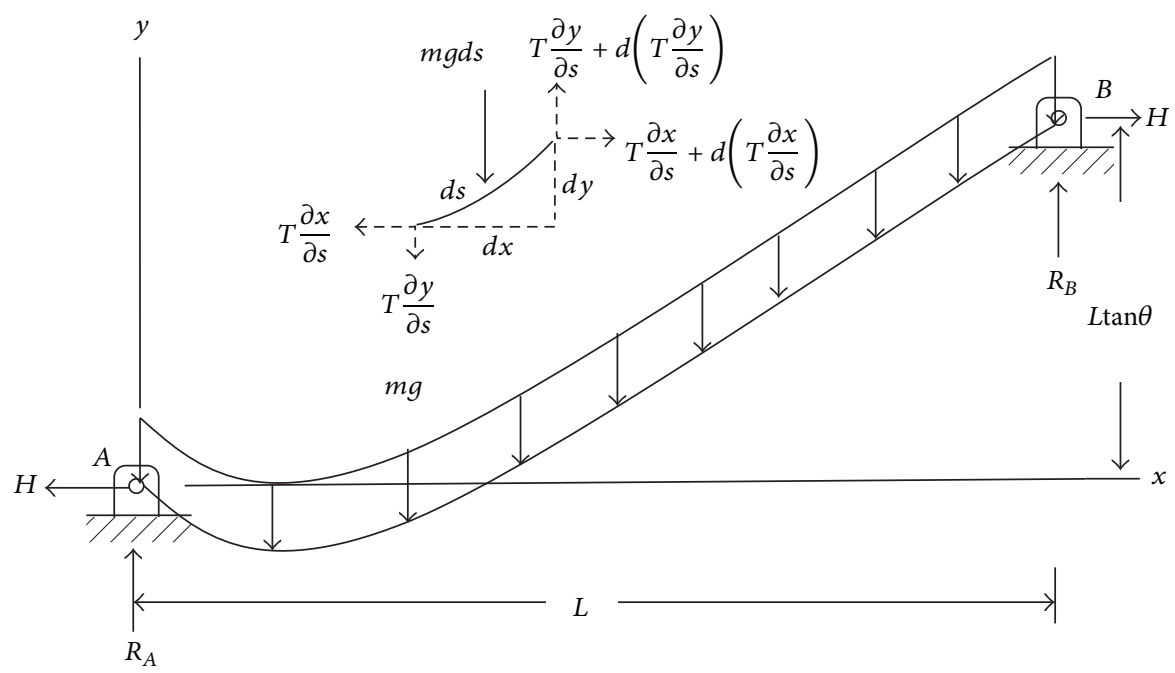

FIGURE 1: Free-body of static equilibrium of catenaries without bending stiffness.

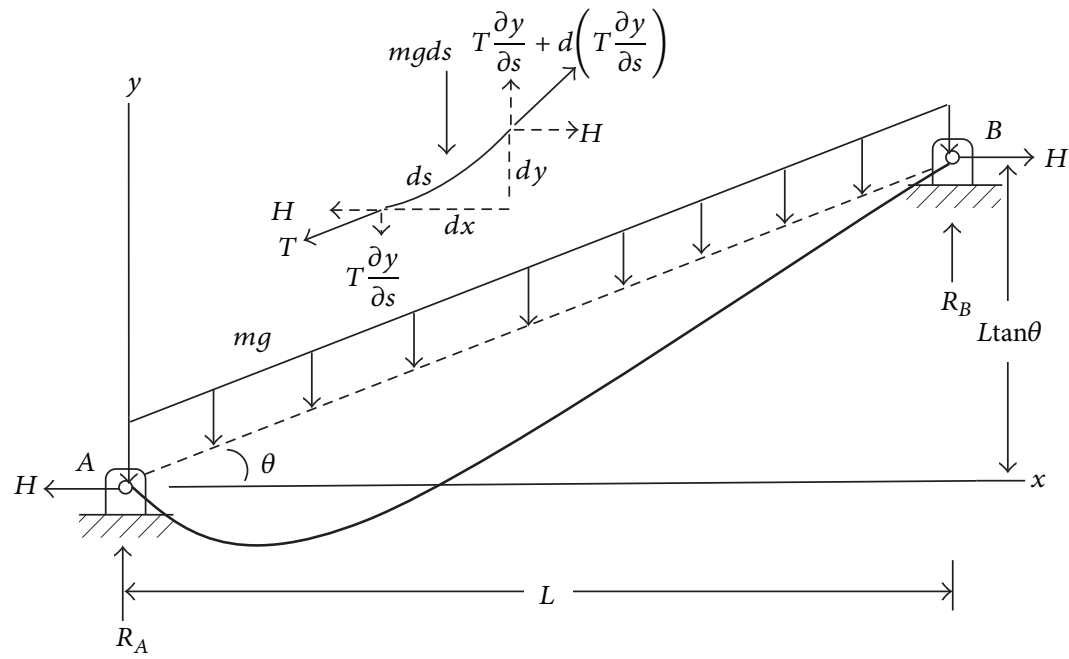

FIGURE 2: Free-body of static equilibrium of a suspended cable with its self-weight distributed uniformly without bending stiffness.

The boundary condition is set to the hinges at the two ends and the solution can be expressed by a parabola. Consider

$$
\bar{y}=\frac{q \sec \theta}{2}\left(\bar{x}^{2}-\bar{x}\right)+\bar{x} \tan \theta .
$$

The two hinges are at the same elevation level $\left(\theta=0^{\circ}\right)$, and (7) is simplified as

$$
\bar{y}=\frac{q}{2}\left(\bar{x}^{2}-\bar{x}\right)
$$

Equation (6) must accept the small sag cable assumption. Most previous studies regarded a ratio of midpoint sag to span below $1 / 8$ as small sag cable $(y(0.5 L) / L<1 / 8, H>m g L)$. On the contrary, a ratio of midpoint sag to span greater than $1 / 8$ is considered as large sag cable $(y(0.5 L) / L>1 / 8, H<m g L)$ [1].

\section{Conventional Solution of the Equation of Static Equilibrium of Catenaries and Parabolas with Bending Stiffness}

3.1. Differential Equation of Catenaries with Bending Stiffness. As shown in Figure 3, the bending moment and shear are incorporated into the catenary. The equation of static equilibrium parallel to the $x$-axis is

$$
\frac{\partial}{\partial s}\left(T \frac{\partial x}{\partial s}+V \frac{\partial y}{\partial s}\right)=0
$$

The horizontal tensile force $H=T(\partial x / \partial s)+V(\partial y / \partial s)$ is a constant. The equation of static equilibrium perpendicular to the $y$-axis is

$$
\frac{\partial}{\partial s}\left(T \frac{\partial y}{\partial s}-V \frac{\partial x}{\partial s}\right)=m g
$$




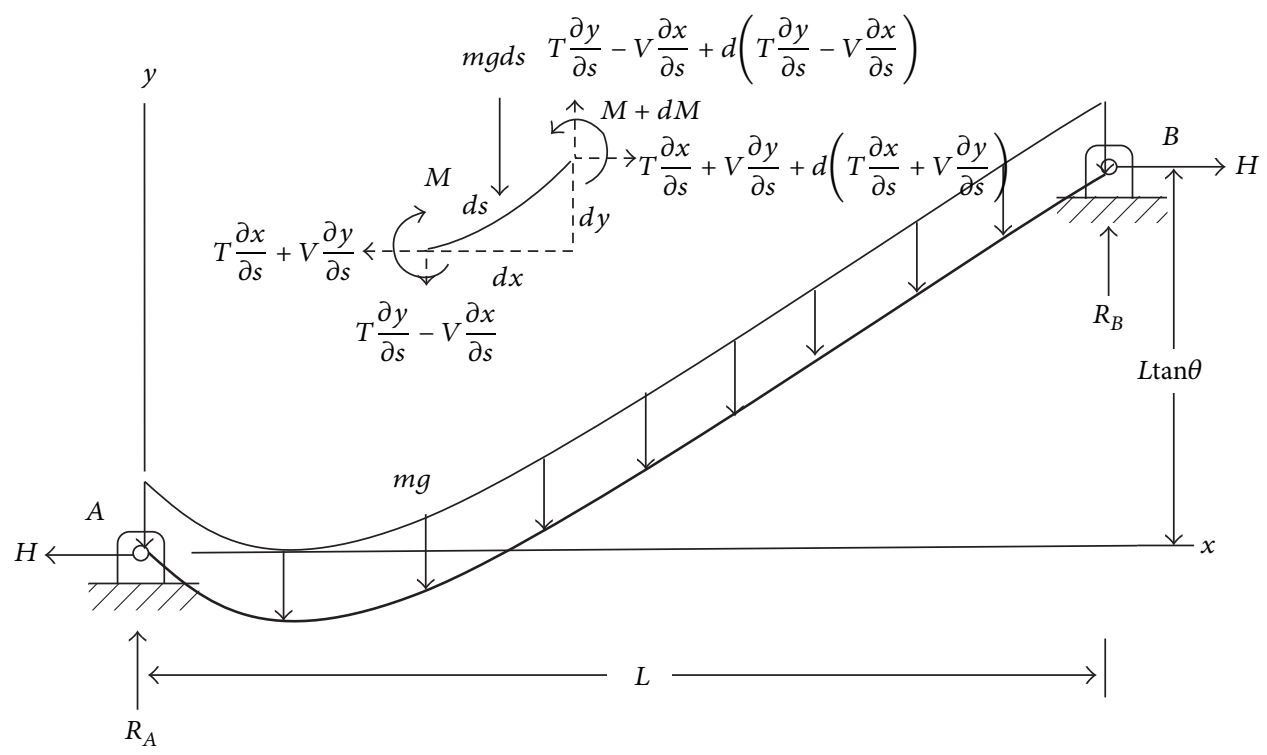

FIGURE 3: Free-body of static equilibrium of catenaries with bending stiffness.

The equilibrium of cable segments yields

$$
V=\frac{\partial M}{\partial s} \text {. }
$$

Compilation yields

$$
H \frac{\partial^{2} y}{\partial x^{2}}-\frac{\partial^{2} M}{\partial x^{2}}=m g \frac{\partial s}{\partial x}
$$

The moment-curvature relation of a large-sag cable is

$$
M=E I \kappa=\frac{E I y^{\prime \prime}}{\left(1+y^{\prime 2}\right)^{3 / 2}} .
$$

$E I$ is flexural rigidity, $E$ the elastic modulus, and $I$ is the second moment of area. In (13), bending moments applied with compression on the upper part of the cable is regarded as positive. By substituting (13) into (12),

$$
H \frac{\partial^{2} y}{\partial x^{2}}-\frac{\partial^{2}}{\partial x^{2}}\left[E I \frac{y^{\prime \prime}}{\left(1+y^{\prime 2}\right)^{3 / 2}}\right]=m g \sqrt{1+y^{\prime 2}} .
$$

3.2. Solution of Parabolas with Bending Stiffness. Due to the difficulty in finding a solution to (14), previous methods could not solve catenaries consisting of bending stiffness and large sag. In the textbook [1], the most common solution is to simplify (12); it is a classical small sag simplified solution using linear curvature. Assuming a tense cable caused by an enormous tensile force, neglect the nonlinear terms in (13) and simplify it as $M=E I y^{\prime \prime}$. Assuming (12) being a parabola, simplify it as

$$
H y^{\prime \prime}-E I y^{\prime \prime \prime \prime}=m g \sec \theta .
$$

Dimensionless parameter $\xi^{2}=H L^{2} / E I$ is introduced to show the influence of bending stiffness. Equation (15) is modified as

$$
\bar{y}^{\prime \prime \prime \prime}-\xi^{2} \bar{y}^{\prime \prime}=-\xi^{2} q \sec \theta
$$

Boundary conditions of hinges $(\bar{y}(0)=0, \bar{y}(1)=\tan \theta$, and $\left.\bar{y}^{\prime \prime}(0)=\bar{y}^{\prime \prime}(1)=0\right)$ are substituted into the solution to (16), yielding a sag equation that includes hinges and excludes bending moments at the two ends. Consider

$$
\begin{aligned}
\bar{y}= & \frac{q \sec \theta}{2}\left(\bar{x}^{2}-\bar{x}\right)+\tan \theta \bar{x} \\
& +\frac{q \sec \theta}{\xi^{2}}\left[1-\cosh (\xi \bar{x})+\tanh \left(\frac{\xi}{2}\right) \sinh (\xi \bar{x})\right] .
\end{aligned}
$$

The two hinges are at the same elevation level $\left(\theta=0^{\circ}\right)$, and (17) is simplified as

$\bar{y}=\frac{q}{2}\left(\bar{x}^{2}-\bar{x}\right)+\frac{q}{\xi^{2}}\left[1-\cosh (\xi \bar{x})+\tanh \left(\frac{\xi}{2}\right) \sinh (\xi \bar{x})\right]$.

Dimensionless bending moment equation of hinges on a horizontal cable is

$$
\begin{aligned}
\frac{\bar{y}^{\prime \prime}}{\xi^{2}} & =\frac{M}{(H L)}=\bar{M} \\
& =\frac{q}{\xi^{2}}\left[1-\cosh (\xi \bar{x})+\tanh \left(\frac{\xi}{2}\right) \sinh (\xi \bar{x})\right] .
\end{aligned}
$$

Bending moments applied with compression on the upper part of the cable are regarded as positive. The substitution of the fixed-end boundary condition shows that the slope of the hinge pivot is not equal to zero but to the slope of the cable $\left(\bar{y}(0)=0, \bar{y}(1)=\tan \theta, \bar{y}^{\prime}(0)=\tan \theta, \bar{y}^{\prime}(1)=\tan \theta\right)$. The obtained dimensionless fixed-end bending moment is

$$
\begin{aligned}
& \bar{M}_{A}=\frac{M_{A}}{(H L)}=q \sec \theta\left[-\frac{1}{\xi^{2}}+\frac{\operatorname{coth}(\xi / 2)}{(2 \xi)}\right], \\
& \bar{M}_{B}=\frac{M_{B}}{(H L)}=q \sec \theta\left[\frac{1}{\xi^{2}}-\frac{\operatorname{coth}(\xi / 2)}{(2 \xi)}\right] .
\end{aligned}
$$




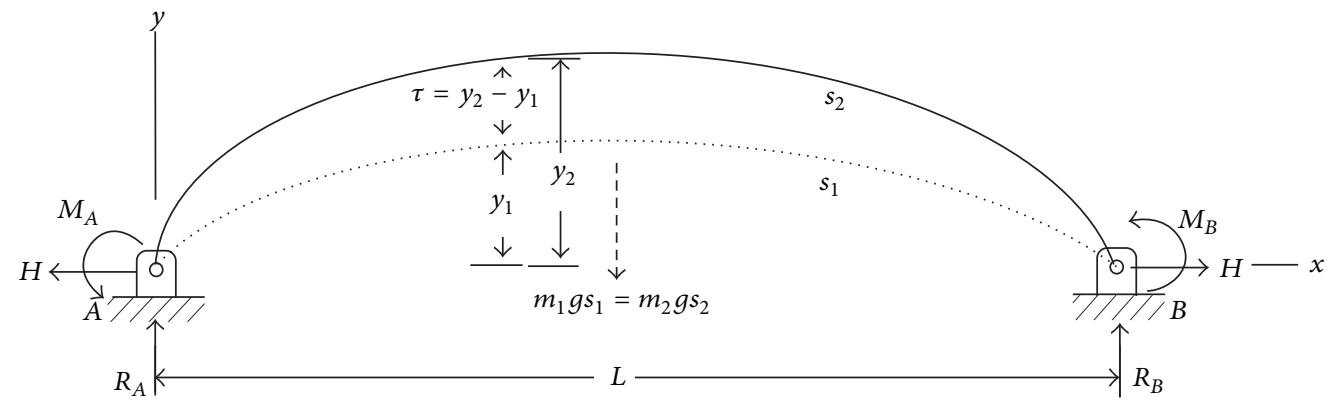

FIgURE 4: The comparison model between number 1 and number 2 catenaries.

$M_{A}$ and $M_{B}$ are bending moments at the anchorage, having positive values when rotating counterclockwise. The sag equation is

$$
\begin{aligned}
\bar{y}= & \frac{q \sec \theta}{2}\left(\bar{x}^{2}-\bar{x}\right)+\tan \theta \bar{x}+\frac{q \sec \theta}{2 \xi} \operatorname{coth}\left(\frac{\xi}{2}\right) \\
& \times\left[1-\cosh (\xi \bar{x})+\tanh \left(\frac{\xi}{2}\right) \sinh (\xi \bar{x})\right] .
\end{aligned}
$$
as

For a horizontal parabola $\left(\theta=0^{\circ}\right),(21)$ can be simplified

$$
\begin{aligned}
\bar{y}= & \frac{q}{2}\left(\bar{x}^{2}-\bar{x}\right)+\frac{q}{2 \xi} \operatorname{coth}\left(\frac{\xi}{2}\right) \\
& \times\left[1-\cosh (\xi \bar{x})+\tanh \left(\frac{\xi}{2}\right) \sinh (\xi \bar{x})\right] .
\end{aligned}
$$

The dimensionless bending moment equation for the fixed-end on a horizontal cable is

$$
\begin{aligned}
\frac{\bar{y}^{\prime \prime}}{\xi^{2}} & =\frac{M}{(H L)}=\bar{M} \\
& =q\left\{\frac{1}{\xi^{2}}+\frac{1}{2 \xi}\left[\sinh (\xi \bar{x})-\operatorname{coth}\left(\frac{\xi}{2}\right) \cosh (\xi \bar{x})\right]\right\} .
\end{aligned}
$$

Equations (17) and (21) are solutions based on parabolas. This is different from the large sag cable approximation in [711].

\section{Novel Catenary Model of Static Equilibrium with Bending Stiffness}

4.1. Novel Catenary Bending Moment Equation. To overcome the difficulty of finding a solution using (14), this study adopted a novel concept to modify (14). Figure 4 is a comparison model between number 1 and number 2 catenaries. Based on (5), number 1 catenary was set to include the tensile force but not the bending moment. Consider

$$
H \frac{\partial^{2} y_{1}}{\partial x^{2}}=m_{1} g \frac{\partial s_{1}}{\partial x}
$$

$m_{1}$ is the per-unity-length mass of number 1 catenary, $s_{1}$ is the length of number 1 catenary curve, and $y_{1}$ is the sag of number 1 catenary.
Based on (12), number 2 catenary was introduced to include the shear and bending moment. Consider

$$
H \frac{\partial^{2} y_{2}}{\partial x^{2}}-\frac{\partial^{2} M}{\partial x^{2}}=m_{2} g \frac{\partial s_{2}}{\partial x} \text {. }
$$

$m_{2}$ is the per-unity-length mass of number 2 catenary, $s_{2}$ is the length of number 2 catenary curve, and $y_{2}$ is the sag of number 2 catenary.

The mechanical relation existing between number 1 and number 2 catenaries was independent and indirect. The two catenaries had two definitely different shapes, $s_{1} \neq s_{2}$, and had different masses along the chord, $m_{1} \neq m_{2}$. However, they shared the following in common: they had the same horizontal distance and vertical elevation between the two anchorages, the same identical total cable self-weight, and the same horizontal tensile force, $H$.

Mathematically, (24) can be subtracted by (25) as follows:

$$
\frac{\partial^{2} M}{\partial x^{2}}=H\left(\frac{\partial^{2} y_{2}}{\partial x^{2}}-\frac{\partial^{2} y_{1}}{\partial x^{2}}\right)-g\left(m_{2} \frac{\partial s_{2}}{\partial x}-m_{1} \frac{\partial s_{1}}{\partial x}\right) \text {. }
$$

By integrating (26),

$$
\frac{\partial M}{\partial x}=H\left(\frac{\partial y_{2}}{\partial x}-\frac{\partial y_{1}}{\partial x}\right)-g\left(m_{2} s_{2}-m_{1} s_{1}\right)+C_{1} .
$$

$m_{2} g s_{2}=m_{1} g s_{1}$ and (27) is modified as

$$
\frac{\partial M}{\partial x}=H\left(\frac{\partial y_{2}}{\partial x}-\frac{\partial y_{1}}{\partial x}\right)+C_{1}
$$

Another integration could obtain the bending moment function, $M$. Consider

$$
M=H\left(y_{2}-y_{1}\right)+C_{1} x+C_{2} .
$$

Equation (29) is a new expression of the cable bending moment equation. The first characteristic allows it to avoid the difficulty of (14), which requires a fourth order differential equation to find the solution; instead, (29) directly finds the sag from the bending moment equation. The second characteristic is that a relation is not needed between sag $y_{2}$ and $y_{1}$ of the number 1 and number 2 catenaries. Simply put, (29) is a mathematical equation that expresses the bending moment of number 2 catenary from the sag of number 1 catenary. The two catenaries happen to have the same self-weight and horizontal tensile force. In other words, any 
form of $y_{1}$ can be selected to calculate the $y_{2}$ with bending stiffness.

Constants of integration $C_{1}$ and $C_{2}$ are obtained based on the boundary conditions. The two catenaries shared the same anchorage elevation, $y_{2}(0)=y_{1}(0)$ and $y_{2}(L)=y_{1}(L)$. $M_{A}$ and $M_{B}$ are the bending moments at the anchorage that show positive value when rotating counterclockwise. Bending moments, when applied with compression on the upper part of the cable, are regarded as positive. The substitution of $M(0)=-M_{A}$ and $M(L)=M_{B}$ results in $C_{1}=\left(M_{A}+M_{B}\right) / L$ and $C_{2}=-M_{A}$. Equation (29) is modified as

$$
M=H\left(y_{2}-y_{1}\right)+M_{A}\left(\frac{x}{L}-1\right)+\frac{M_{B} x}{L} .
$$

The dimensionless bending moment equation is

$$
\frac{M}{(H L)}=\bar{M}=\bar{y}_{2}-\bar{y}_{1}+\bar{M}_{A}(\bar{x}-1)+\bar{M}_{B} \bar{x} .
$$

Equation (30) can also be easily derived by examining the static equilibrium of the free-body diagram of number 1 and number 2 catenaries.

4.2. Verification of the Correctness of the Novel Bending Moment Equation Using the Parabola. This study used the parabola to verify (31). Let (7) be $\bar{y}_{1}, \bar{y}_{1}^{\prime \prime}=q \sec \theta$. By substituting $\bar{y}_{1}^{\prime \prime}=q \sec \theta$ and $M=E I y_{2}^{\prime \prime}$ into (31),

$$
\bar{y}_{2}^{\prime \prime}=\xi^{2}\left(\bar{y}_{2}-\bar{y}_{1}\right)+\xi^{2} \bar{M}_{A}(\bar{x}-1)+\xi^{2} \bar{M}_{B} \bar{x} .
$$

The correctness of (29) can be verified with $\bar{y}_{2}$ (which is (21)), a solution obtained by substituting the fixed-end boundary condition.

4.3. Novel Catenary Differential Equation. The momentcurvature relation of the large sag catenary is $M=E I y^{\prime \prime} /(1+$ $\left.y^{\prime 2}\right)^{3 / 2}$, and nonlinear terms are not neglected. Parameter $\tau$ is set to

$$
\tau=\bar{y}_{2}-\bar{y}_{1} \text {. }
$$

$\tau$ represents difference of sag between number 1 and number 2 catenaries caused by bending moments. $\bar{y}_{2}=\bar{y}_{1}+\tau$ and $\bar{y}_{2}^{\prime \prime}=\bar{y}_{1}^{\prime \prime}+\tau^{\prime \prime}$. By substituting the results into (31) to obtain the differential equation of large sag catenary,

$$
\begin{aligned}
\frac{\bar{y}_{2}^{\prime \prime}}{\left(1+\bar{y}_{2}^{\prime 2}\right)^{3 / 2}} & =\frac{\bar{y}_{1}^{\prime \prime}+\tau^{\prime \prime}}{\left(1+\bar{y}_{2}^{\prime 2}\right)^{3 / 2}} \\
& =\xi^{2}\left(\bar{y}_{2}-\bar{y}_{1}\right)+\xi^{2} \bar{M}_{A}(\bar{x}-1)+\xi^{2} \bar{M}_{B} \bar{x}
\end{aligned}
$$

Given that (4) is $\bar{y}_{1}, \bar{y}_{1}^{\prime}=\sinh (q \bar{x}+a-q / 2)$ and $\bar{y}_{1}^{\prime \prime}=$ $q \cosh (q \bar{x}+a-q / 2)$. In a cable-stayed bridge, $95 \%$ of the cables have $\xi \geq 50$ [13]. $\left(1+\bar{y}_{2}^{\prime 2}\right)^{3 / 2} \approx\left(1+\bar{y}_{1}^{\prime 2}\right)^{3 / 2}$, and $\left(1+\bar{y}_{2}^{\prime 2}\right)^{3 / 2}$ can be replaced by $\left(1+\bar{y}_{1}^{\prime 2}\right)^{3 / 2}=\left[1+\sinh ^{2}(q \bar{x}+a-q / 2)\right]^{3 / 2}=$ $\cosh ^{3}(q \bar{x}+a-q / 2)$. Subsequently, (34) becomes the differential equation with a bending moment sag of $\tau$. Consider

$$
\begin{aligned}
\frac{\tau^{\prime \prime}}{\xi^{2}}- & \cosh ^{3}\left(q \bar{x}-\frac{q}{2}\right) \tau \\
= & -\frac{q}{\xi^{2}} \cosh \left(q \bar{x}-\frac{q}{2}\right) \\
& +\cosh ^{3}\left(q \bar{x}-\frac{q}{2}\right)\left[\bar{M}_{A}(\bar{x}-1)+\bar{M}_{B} \bar{x}\right] .
\end{aligned}
$$

The large horizontal tensile force and small bending stiffness make $1 / \xi^{2}$ much smaller as compared to other parameters. The $\tau^{\prime \prime}$ in (35) is multiplied by small parameter $1 / \xi^{2}$ to form a paradigmatic boundary layer problem that involves the multiplication of a highest order derivative with small parameter $1 / \xi^{2}$. This implies that an enormous function variation exists in the differential region of the catenary anchorage. Leaving the differential region, function variations quickly come to a mild plane and maintain at a steady state. The division that signifies the rapid and drastic function variation is known as the boundary layer and the differential region at the catenary anchorage is known as the thickness of the boundary layer. Since (35) did not have an analytical solution, this study adopted the WKB method in the perturbation method to find the approximation solution.

\section{WKB Catenary Solution with Bending Stiffness}

First, the homogeneous solution of (35) was found as follows:

$$
\frac{\tau^{\prime \prime}}{\xi^{2}}-\cosh ^{3}\left(q \bar{x}+a-\frac{q}{2}\right) \tau=0
$$

The WKB approximation method developed by Wentzel, Kramers, and Brillouin [2-4] was adopted to find the solution to (36).

In the classical Sturm-Liouville equation [5], $\tau^{\prime \prime} / \xi^{2}+$ $q_{1}(x) \tau=0$. When $q_{1}(x)>0$, the first order approximation is $\tau=\left[C_{1} \sin \left(\xi \int \sqrt{q_{1}} d x\right)+C_{2} \cos \left(\xi \int \sqrt{q_{1}} d x\right)\right] / \sqrt[4]{q_{1}}$. When $q_{1}(x)<0$, the first order approximation is $\tau=$ $\left[C_{1} \sinh \left(\xi \int \sqrt{q_{1}} d x\right)+C_{2} \cosh \left(\xi \int \sqrt{q_{1}} d x\right)\right] / \sqrt[4]{q_{1}}$.

Equation (36) is $q_{1}=-\cosh ^{3}(q \bar{x}+a-q / 2)<0$, and the $\mathrm{WKB}$ approximation is

$$
\tau_{h}=\frac{C_{1} \sinh \left[\xi \int \cosh ^{3 / 2}(q \bar{x}+a-q / 2) d x\right]+C_{2} \cosh \left[\xi \int \cosh ^{3 / 2}(q \bar{x}+a-q / 2) d x\right]}{\cosh ^{3 / 4}(q \bar{x}+a-q / 2)} .
$$


The result of $\xi \int \cosh ^{3 / 2}(q \bar{x}+a-q / 2) d x$ in (37) is

$$
\begin{aligned}
\delta=\xi \int \cosh ^{3 / 2}\left(q \bar{x}+a-\frac{q}{2}\right) d x \\
=\frac{2 \xi}{3 q}\left\{\sqrt{\cosh \left(q \bar{x}+a-\frac{q}{2}\right)} \sinh \left(q \bar{x}+a-\frac{q}{2}\right)\right. \\
\\
\quad-i F(\phi \mid 2)\} .
\end{aligned}
$$

$F(\phi \mid 2)$ is the elliptic integral of the first kind, $\phi=i(q \bar{x}+a-$ $q / 2) / 2$, and $i$ is the imaginary unit. Consider

$$
\begin{aligned}
F(\phi \mid 2) & =\int_{0}^{\phi} \frac{1}{\sqrt{1-2 \sin ^{2} \phi}} d \phi \\
& =\int \frac{i q}{2 \sqrt{1+2 \sinh ^{2}[(q \bar{x}+a-q / 2) / 2]}} d x .
\end{aligned}
$$

$1 / \xi^{2}$ is too small and therefore the nonhomogeneous solution could be obtained by neglecting $\tau^{\prime \prime} / \xi^{2}$ in (36). Consider

$$
\begin{aligned}
&-\cosh ^{3}\left(q \bar{x}-\frac{q}{2}\right) \tau_{p}=-\frac{q}{\xi^{2}} \cosh \left(q \bar{x}-\frac{q}{2}\right) \\
&+\cosh ^{3}\left(q \bar{x}-\frac{q}{2}\right) \\
& \times\left[\bar{M}_{A}(\bar{x}-1)+\bar{M}_{B} \bar{x}\right], \\
& \tau_{p}=\frac{q}{\xi^{2}} \operatorname{sech}^{2}\left(q \bar{x}-\frac{q}{2}\right)-\bar{M}_{A}(\bar{x}-1)-\bar{M}_{B} \bar{x} .
\end{aligned}
$$

The final result is $\tau=\tau_{h}+\tau_{p}$. Define

$$
\begin{aligned}
\tau= & \frac{C_{1} \sinh \delta}{\cosh ^{3 / 4}(q \bar{x}+a-q / 2)}+\frac{C_{2} \cosh \delta}{\cosh ^{3 / 4}(q \bar{x}+a-q / 2)} \\
& +\frac{q}{\xi^{2}} \operatorname{sech}^{2}\left(q \bar{x}+a-\frac{q}{2}\right)-\bar{M}_{A}(\bar{x}-1)-\bar{M}_{B} \bar{x} .
\end{aligned}
$$

The total sag is $\bar{y}=\bar{y}_{2}=\bar{y}_{1}+\tau$. Consider

$$
\begin{aligned}
\bar{y}= & \frac{1}{q}\left\{\cosh \left(q \bar{x}+a-\frac{q}{2}\right)-\cosh \left(a-\frac{q}{2}\right)\right\} \\
& +\frac{C_{1} \sinh \delta}{\cosh ^{3 / 4}(q \bar{x}+a-q / 2)}+\frac{C_{2} \cosh \delta}{\cosh ^{3 / 4}(q \bar{x}+a-q / 2)} \\
& +\frac{q}{\xi^{2}} \operatorname{sech}^{2}\left(q \bar{x}+a-\frac{q}{2}\right)-\bar{M}_{A}(\bar{x}-1)-\bar{M}_{B} \bar{x} .
\end{aligned}
$$

For a horizontal catenary $\left(\theta=0^{\circ}, a=0\right),(41)$ can be simplified as

$$
\begin{aligned}
\tau= & \frac{C_{3} \sinh \delta}{\cosh ^{3 / 4}(q \bar{x}-q / 2)}+\frac{C_{4} \cosh \delta}{\cosh ^{3 / 4}(q \bar{x}-q / 2)} \\
& +\frac{q}{\xi^{2}} \operatorname{sech}^{2}\left(q \bar{x}-\frac{q}{2}\right)-\bar{M}_{A}(\bar{x}-1)-\bar{M}_{B} \bar{x} .
\end{aligned}
$$

$\delta=\{(2 \xi / 3 q)[\sqrt{\cosh (q \bar{x}-q / 2)} \sinh (q \bar{x}-q / 2)-i F(\phi \mid 2)]\}$. The total sag is

$$
\begin{aligned}
\bar{y}= & \frac{1}{q}\left\{\cosh \left[q\left(\bar{x}-\frac{1}{2}\right)\right]-\cosh \left(\frac{q}{2}\right)\right\} \\
& +\frac{C_{3} \sinh \delta}{\cosh ^{3 / 4}(q \bar{x}-q / 2)}+\frac{C_{4} \cosh \delta}{\cosh ^{3 / 4}(q \bar{x}-q / 2)} \\
& +\frac{q}{\xi^{2}} \operatorname{sech}^{2}\left(q \bar{x}-\frac{q}{2}\right)-\bar{M}_{A}(\bar{x}-1)-\bar{M}_{B} \bar{x} .
\end{aligned}
$$

A solution could be found by substituting (42) and (44) into the boundary conditions. The advantage of the horizontal catenaries in (44) is their arbitrary abilities to be substituted into any desired boundary conditions. When the catenaries are hinges and do not possess bending moments, $\bar{M}_{B}=\bar{M}_{A}=0$. Considering that $\bar{y}(0)=0$ and $\bar{y}(1)=0$, let $\delta_{1}=(2 \xi / 3 q)[\sqrt{\cosh (q / 2)} \sinh (q / 2)-i F(i q / 4 \mid 2)]$ and simplify the solution to yield

$$
C_{3}=0, \quad C_{4}=-\frac{q \operatorname{sech} \delta_{1}}{\xi^{2} \cosh ^{5 / 4}(q / 2)} .
$$

The substitution of (45) into (43) generates the bending moment sag equation for horizontal hinged catenaries as follows:

$$
\begin{aligned}
\tau=\frac{q}{\xi^{2}}[ & \operatorname{sech}^{2}\left(q \bar{x}-\frac{q}{2}\right) \\
& \left.-\frac{\operatorname{sech} \delta_{1} \cosh \delta}{\cosh ^{5 / 4}(q / 2) \cosh ^{3 / 4}(q \bar{x}-q / 2)}\right] .
\end{aligned}
$$

The substitution of (45) into (44) generates the total sag equation for horizontal hinged catenaries as follows:

$$
\begin{aligned}
& \bar{y}= \frac{1}{q}\left\{\cosh \left[q\left(\bar{x}-\frac{1}{2}\right)\right]-\cosh \left(\frac{q}{2}\right)\right\} \\
&+\frac{q}{\xi^{2}}\left[\operatorname{sech}^{2}\left(q \bar{x}-\frac{q}{2}\right)\right. \\
&\left.\quad-\frac{\operatorname{sech} \delta_{1} \cosh \delta}{\cosh ^{5 / 4}(q / 2) \cosh ^{3 / 4}(q \bar{x}-q / 2)}\right] .
\end{aligned}
$$

Boundary conditions of fixed-ends $(\bar{y}(0)=0, \bar{y}(1)=0$, $\bar{y}^{\prime}(0)=0, \bar{y}^{\prime}(1)=0$ ) are substituted into the solution to (47), and the obtained constants of integration are

$$
C_{3}=0 \text {, }
$$

$\mathrm{C}_{4}$

$$
=-\frac{4 \sinh (q / 2) \operatorname{sech} \delta_{1}\left[\xi^{2} \cosh ^{3}(q / 2)-2 q^{2}\right]}{\xi^{2} \cosh ^{5 / 4}(q / 2)\left[4 \xi \cosh ^{5 / 2}(q / 2) \tanh \delta_{1}-3 q \sinh (q / 2)\right]} .
$$

$\bar{M}_{B}=-\bar{M}_{A}$, and the bending moment, $\bar{M}_{A}$, of the left fixedend is 


$$
\bar{M}_{A}=\frac{\operatorname{sech}^{2}(q / 2)\left\{\sinh (q / 2)\left[4 \xi^{2} \cosh ^{3}(q / 2)-5 q^{2}\right]-4 q \xi \cosh ^{5 / 2}(q / 2) \tanh \left(\delta_{1}\right)\right\}}{\xi^{2}\left[4 \xi \cosh ^{5 / 2}(q / 2) \tanh \left(\delta_{1}\right)-3 q \sinh (q / 2)\right]} .
$$

Substitute (48) and (49) into (43) to obtain the bending moment sag equation for horizontal fixed-end catenaries as follows:

$$
\tau=\frac{C_{4} \cosh \delta}{\cosh ^{3 / 4}(q \bar{x}-q / 2)}+\frac{q}{\xi^{2}} \operatorname{sech}^{2}\left(q \bar{x}-\frac{q}{2}\right)+\bar{M}_{A} .
$$

Substitute (48) and (49) into (44) to obtain the total sag equation for horizontal fixed-end catenaries as follows:

$$
\begin{aligned}
\bar{y}= & \frac{1}{q}\left\{\cosh \left[q\left(\bar{x}-\frac{1}{2}\right)\right]-\cosh \left(\frac{q}{2}\right)\right\} \\
& +\frac{C_{4} \cosh \delta}{\cosh ^{3 / 4}(q \bar{x}-q / 2)}+\frac{q}{\xi^{2}} \operatorname{sech}^{2}\left(q \bar{x}-\frac{q}{2}\right)+\bar{M}_{A} .
\end{aligned}
$$

Substitute (43) into (31) to obtain the bending moment equation of horizontal fixed-end catenaries as follows:

$$
\bar{M}=\frac{C_{4} \cosh \delta}{\cosh ^{3 / 4}(q \bar{x}-q / 2)}+\frac{q}{\xi^{2}} \operatorname{sech}^{2}\left(q \bar{x}-\frac{q}{2}\right) .
$$

$C_{4}$ is illustrated in (45) and (48). Without the necessity of a general perturbation method $[10,11]$ that requires the matching of cable and beam segments, the proposed equations (44) and (52) can describe the drastic function variations within and without the boundary layer with a single equation.

\section{Results and Discussion}

Figure 5 shows a comparison between the total sag of parabolas and catenaries. The figure indicates that under any $q$ and $\xi$, the WKB solution of catenaries derived from (51) was between (5) and (22). At the same $q$ (1.0), the three solutions demonstrated greater differences as $\xi$ reduced. As $q=1.0$ and $\xi=20$, the total sag retrieved by (51) of the cable span midpoint was -0.105 . This corresponds to [12]. At the same $\xi(\xi=40)$, the three solutions demonstrated greater differences as $q$ increased. When $q=0.5,(22)$ shows a good approximation of (51).

Fundamental mechanics show that the difference between (5) and (8) increased with $q$. Therefore, when discussing the influence of $\xi$ on sag, the sag of (5) and (8) should be divided by (51) and (22) to compare with sag $\tau$ caused by the bending moment. The bending moment sag, $\tau$, corresponds to the solution of (22) that contains a bending stiffness parabola. Consider

$$
\tau=\frac{q}{2 \xi} \operatorname{coth}\left(\frac{\xi}{2}\right)\left[1-\cosh (\xi \bar{x})+\tanh \left(\frac{\xi}{2}\right) \sinh (\xi \bar{x})\right] .
$$

First the influence of bending moment sag, $\tau$, on catenaries is evaluated.

Define the ratio of span midpoint bending moment sag to hinged catenaries corresponding to division of (5) and (46). Equation (5) is the sag of the catenary $(\bar{x}=0.5)$, and (46) is the bending moment sag equation for horizontal hinged catenaries $(\bar{x}=0.5)$. Consider

$$
r_{1}=\left(\frac{q}{\xi}\right)^{2} \frac{1-\operatorname{sech} \delta_{1} / \cosh ^{5 / 4}(q / 2)}{\cosh (q / 2)-1} .
$$

$r_{1}$ is the ratio of span midpoint bending moment sag to hinged catenaries.

Under four different $q$ 's $(0.5,1,2$, and 3), Figure 6 views the ratio of span midpoint bending moment sag $\tau$ to catenaries supported by a hinge. The ratio was found to drastically fluctuate between $10 \leq \xi \leq 30$. At all the $q$ values, $\tau$ occupied a higher ratio when $\xi$ was smaller, a ratio that amounted to $7.86 \%$; $\tau$ occupied a smaller ratio when $\xi$ was larger. When $\xi=20$, the ratio was only $1.7 \%$, and when $\xi=50$ the ratio was only $0.3 \%$. Bending stiffness had effective influences when $\xi<20$ in the hinged catenary.

Define the ratio of span midpoint bending moment sag to fixed-end catenaries corresponding to division of (5) and (50). Equation (50) is the bending moment sag equation for horizontal fixed-end catenaries $(\bar{x}=0.5)$. Consider

$$
r_{2}=\frac{q\left(C_{4}+q / \xi^{2}+\bar{M}_{A}\right)}{\cosh (q / 2)-1} .
$$

$r_{2}$ is the ratio of span midpoint bending moment sag to fixed-end catenaries. $C_{4}$ is illustrated in (48). $\bar{M}_{A}$ is illustrated in (49).

Figure 7 demonstrates the fixed-end catenary sag ratio occupied by span midpoint bending moment sag $\tau$. The figure showed a variation trend identical to Figure 6 but with a greatly enhanced value. When $q=1.0, \xi=10$ had a $35.9 \%$ ratio, $\xi=50$ had a $6.9 \%$ ratio, and $\xi=100$ had a $3.4 \%$ ratio. Obviously whether the boundary condition is hinge or fixedend results in significant differences. Fixed-end cables should take bending stiffness influences into account.

Define the ratio of span midpoint bending moment sag of parabolas to catenaries corresponding to division of (50) and (53). Equation (53) is the bending moment sag equation for horizontal fixed-end parabolas $(\bar{x}=0.5)$. Consider

$$
r_{3}=\frac{(q / \xi) \operatorname{csch}(\xi / 2) \sinh ^{2}(\xi / 4)}{C_{4}+q / \xi^{2}+\bar{M}_{A}} .
$$



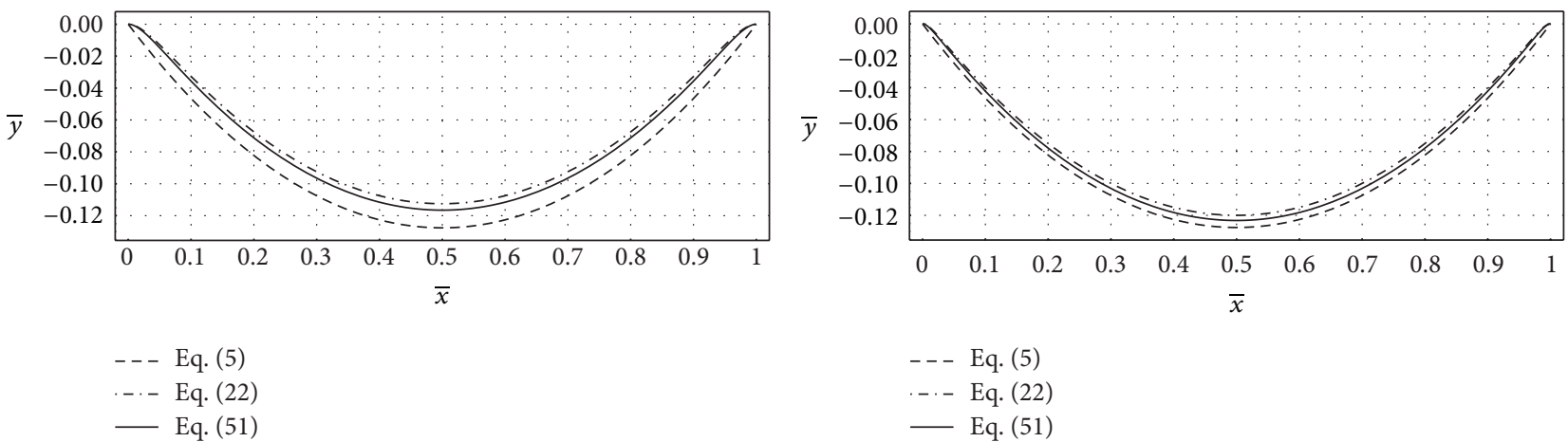

(a) $q=1.0 \xi=40$
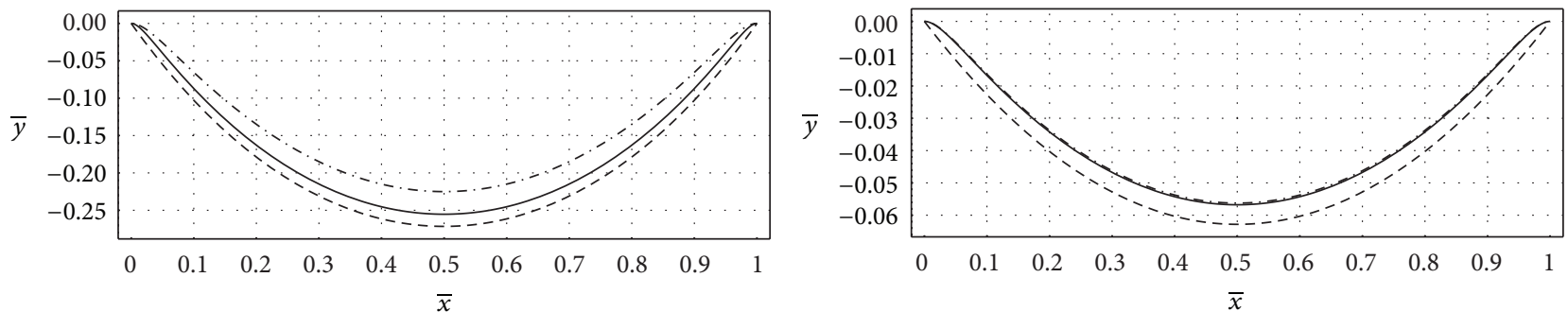

- - Eq. (5)

- - Eq. (5)

-. - Eq. (22)

-. - Eq. (22)

- Eq. (51)

- Eq. $(51)$

(c) $q=2.0 \quad \xi=40$

(d) $q=0.5 \xi=40$

Figure 5: Comparisons between the total sag of parabolas and catenaries.

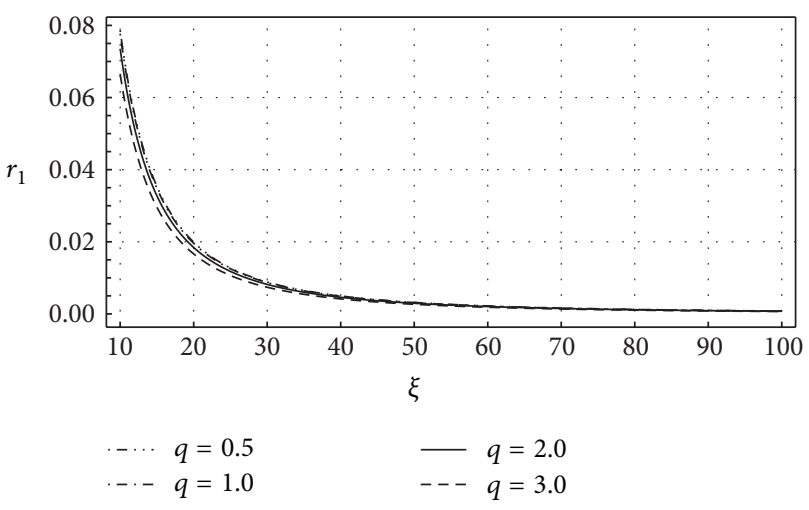

FIGURE 6: The ratio of span midpoint bending moment sag to hinged catenaries $(\bar{x}=0.5)$.

$r_{3}$ is the ratio of span midpoint bending moment sag of parabolas to catenaries. $C_{4}$ is illustrated in (48). $\bar{M}_{A}$ is illustrated in (49).

The ratio differences between bending stiffness and solutions of parabolas and catenaries were compared using (50) and (53). Figure 8 signifies that the ratio varied with $\xi$ under the four different $q$ values $(0.5,1,2$, and 3$)$. The parabola solution was not applicable because a large $q$ led to increased differences; on the contrary, a smaller $q$ led to reduced differences and a parabola solution could replace a catenary

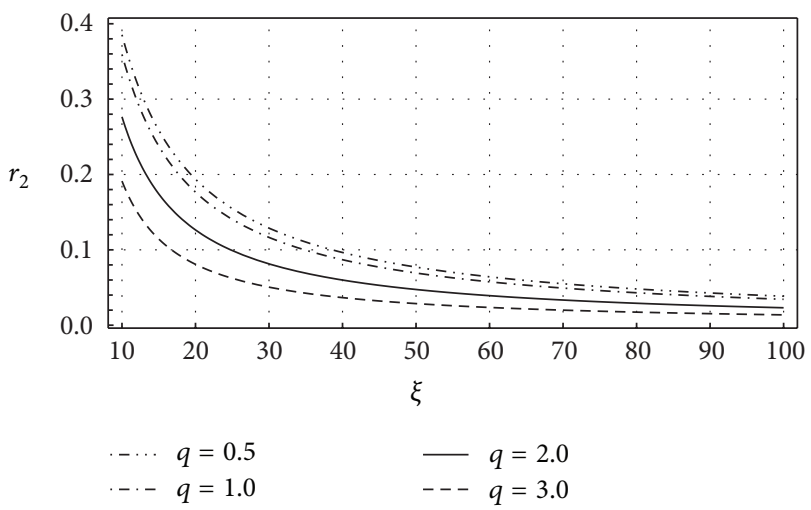

FIGURE 7: The ratio of span midpoint bending moment sag to fixedend catenaries $(\bar{x}=0.5)$.

solution. When $q=0.5$, the ratio varied between $101.9 \%$ and $103.5 \%$. Equation (22) was a good approximation of (51). When $q=1.0$, the ratio varied between $107.6 \%$ and $114 \%$. When $q=3.0$ the ratio could be as high as $242.7 \%$ and (22) was an inappropriate selection.

Figure 9 shows the comparison between the parabola and catenary fixed-end bending moments. As (20) was based on a small sag linear hypothesis, $\bar{M}_{A}$ varied linearly with $q$. However, (49) varied nonlinearly. 


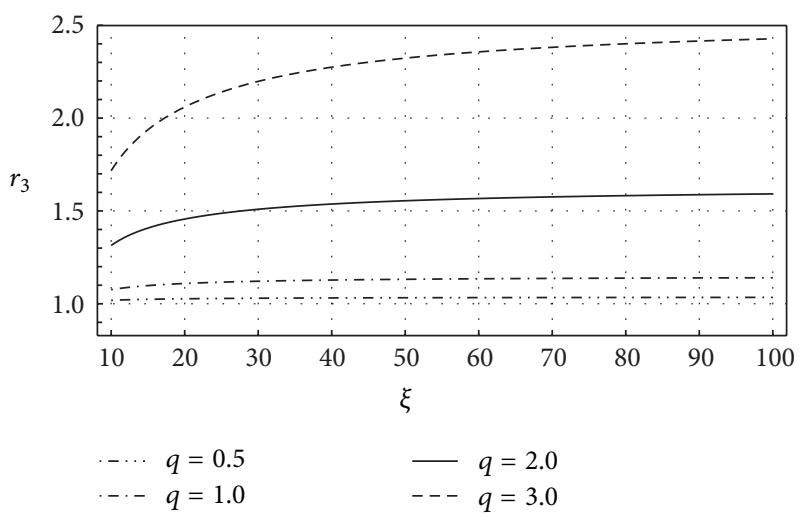

FIGURE 8: The ratio differences between bending stiffness and solutions of parabolas and catenaries.

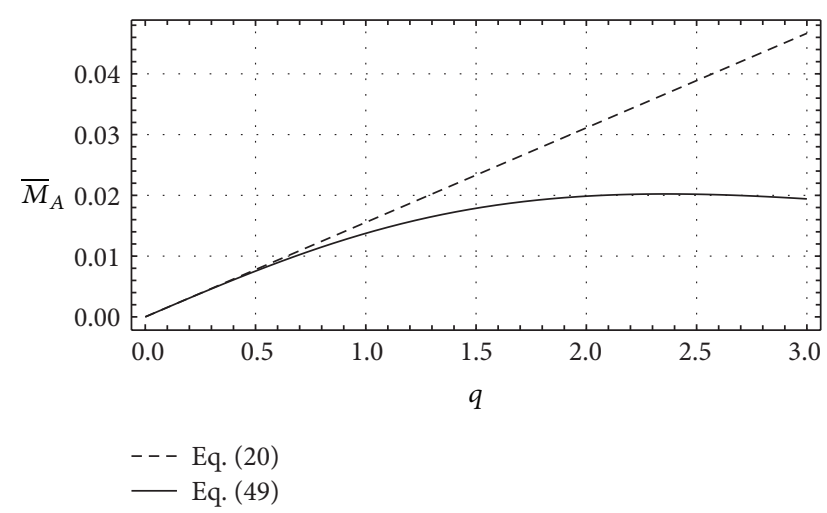

FIGURE 9: The comparison between the parabola and catenary fixedend bending moments $(\xi=30)$.

Define the ratio of fixed-end bending moments of parabolas to catenaries corresponding to division of (20) and (49). Consider

$$
r_{4}=\frac{q \sec \theta\left[-1 / \xi^{2}+\operatorname{coth}(\xi / 2) /(2 \xi)\right]}{\bar{M}_{A}} .
$$

$r_{4}$ is the ratio of fixed-end bending moments of parabolas to catenaries. $\bar{M}_{A}$ is illustrated in (49).

Figure 10 adopts (20) and (49) to evaluate the ratios of influences of bending stiffness on fixed-end bending moments. The figure showed a variation trend extremely similar to Figure 8 . When $q=0.5$, the ratio varied between $102.5 \%$ and $103.5 \%$, and (20) was a good approximation of (49). When $q \leq 0.5$, the parabola solution demonstrated that ratio differences increased with $q$, and when $q=1.0$, the ratio fluctuated between $110.1 \%$ and $114.3 \%$. When $q=3.0$, the ratio could be as high as $250 \%$, and the selection of (20) was an inappropriate choice.

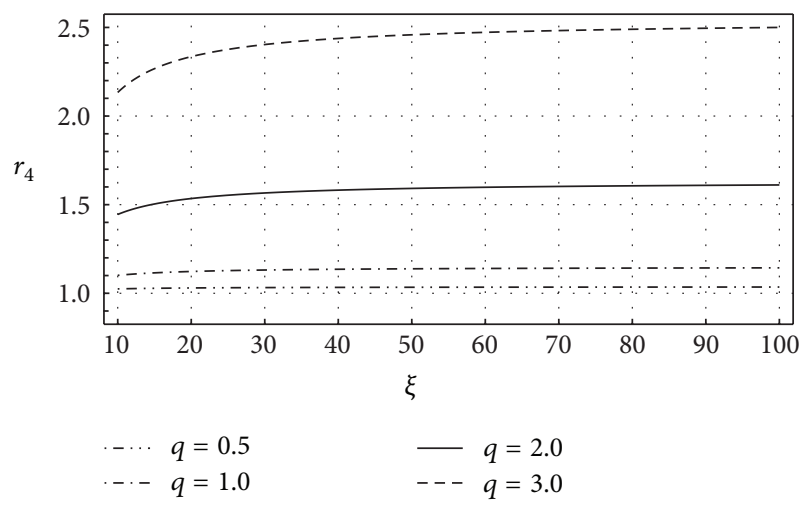

FIGURE 10: The ratios of influences of bending stiffness on fixed-end bending moments.

Figure 11 shows the distribution of parabola and catenary bending moments. The figure indicates that $\xi$ changed the boundary layer thickness and $\bar{M}_{A}$. At the same $q(q=1.0)$, increased $\xi$ and reduced $\bar{M}_{A}$. This resulted in a reduced thickness of boundary layers and a more drastic variation in bending moment functions. $q$ increased the difference between (23) and (52). When $q=0.5$, the catenary solution could be replaced by the parabola solution. In addition, the two solutions of $q=3.0$ showed significant difference in values and shapes and in such a circumstance the parabola was not applicable.

\section{Conclusions}

(1) This study proposed a novel large sag catenary bending moment expression that allows finding the sag directly from the bending moment equation and finding the WKB catenary solution using the WKB method. The matching of cable and beam segments is no longer necessary, thereby overcoming the complexity of boundary layers. Moreover, with only a single equation, the proposed method could simultaneously describe the drastic function variations inside and outside the boundary layer. The method provides a simple calculation of cables with bending stiffness and large sag and fulfills the engineering needs for tensile cable fatigue stress analysis and estimation of suspension cable sag for bridge hoisting.

(2) Statistical analysis revealed a significantly different influence on the behavior of cables with bending stiffness given that the boundary condition was hinges or fixed-ends. Bending stiffness was regarded as effective when $\xi<20$ for the hinged catenary. However, the fixed-end catenary should always consider the influence of bending stiffness. When catenary $q \leq 0.5$, the horizontal tensile force was massive $(H \geq 2 m g L)$. The parabola shows a very good approximation of catenary. It could replace the WKB catenary solution. In addition, $\xi$ had the ability to change the boundary layer thickness where bending moments were dis- 


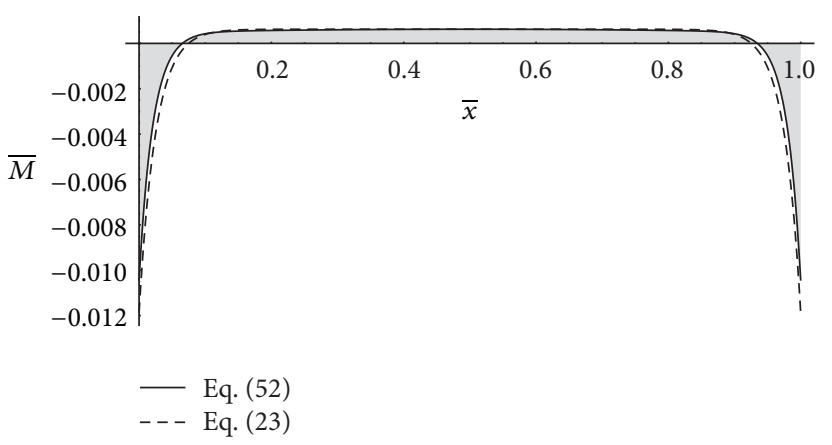

(a) $q=1.0 \xi=40$

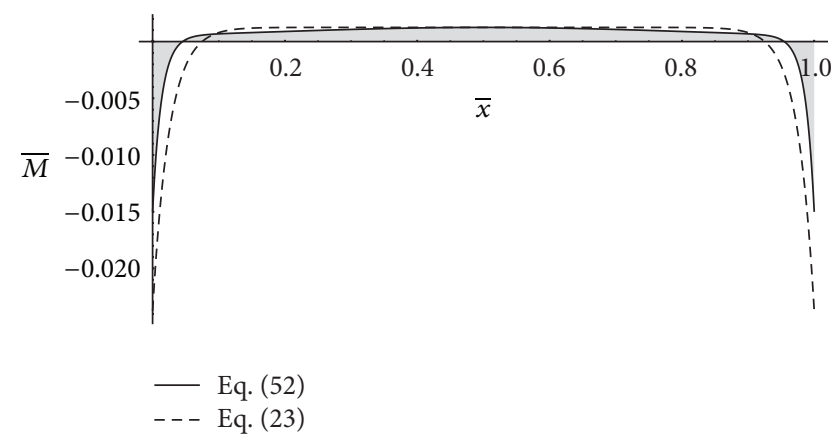

(c) $q=2.0 \xi=40$

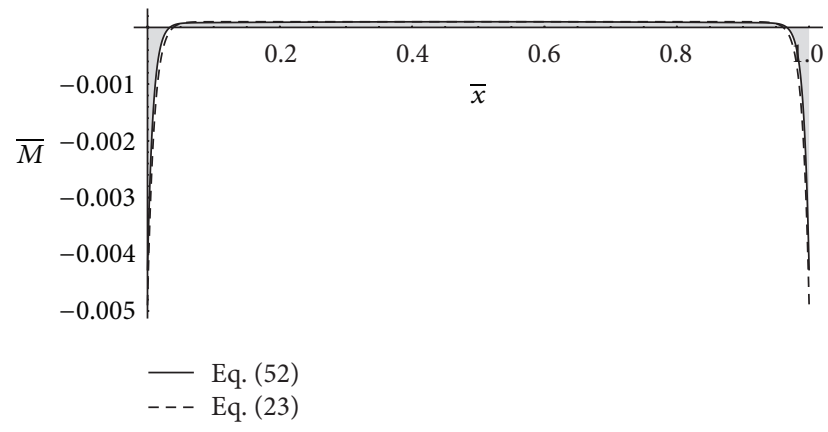

(b) $q=1.0 \xi=100$

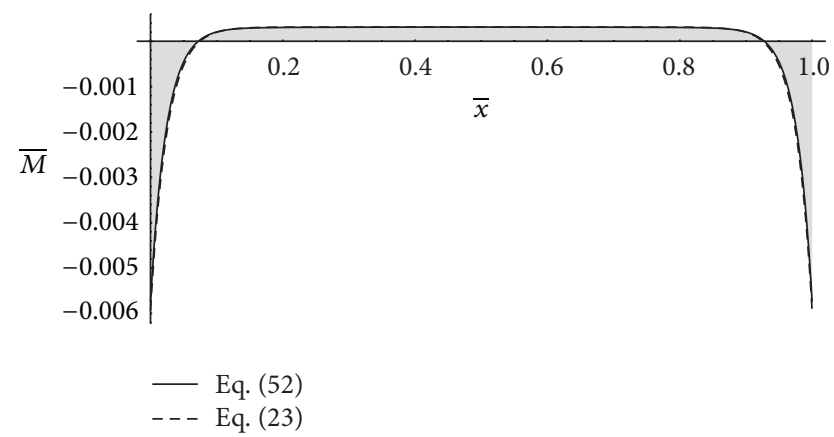

(d) $q=0.5 \xi=40$

FIGURE 11: The distribution of parabola and catenary bending moments.

tributed and fixed-end bending moment. Higher $\xi$ lowered the fixed-end bending moment, reduced the boundary layer, and increased the drastic variation of the bending moment functions.

\section{Conflict of Interests}

The authors declare that there is no conflict of interests regarding the publication of this paper.

\section{References}

[1] H. M. Irvine, Cable Structures, Dover Publications, 1992.

[2] J. J. Burgess, "Bending stiffness in a simulation of undersea cable deployment," International Journal of Offshore and Polar Engineering, vol. 3, no. 3, pp. 197-204, 1993.

[3] E. J. Hinch, Perturbation Methods, Cambridge University Press, Cambridge, UK, 1991.

[4] J. Kevorkian and J. D. Cole, Multiple Scale and Singular Perturbation Methods, Springer, Berlin, Germany, 1996.

[5] A. H. Nayfeh, Introduction to Perturbation Techniques, John Wiley \& Sons, New York, NY, USA, 2011.

[6] M. S. Triantafyllou and G. S. Triantafyllou, "The paradox of the hanging string: an explanation using singular perturbations," Journal of Sound and Vibration, vol. 148, no. 2, pp. 343-351, 1991.

[7] P. Wolfe, "The effect of bending stiffness on inextensible cables," International Journal of Engineering Science, vol. 30, no. 9, pp. 1187-1192, 1992.

[8] H. M. Irvine, "Local bending stresses in cables," International Journal of Offshore and Polar Engineering, vol. 3, no. 3, pp. 172175, 1993.
[9] D. M. Stump and W. B. Fraser, "Bending boundary layers in a moving strip," Nonlinear Dynamics, vol. 21, no. 1, pp. 55-70, 2000

[10] D. M. Stump and G. H. M. van der Heijden, "Matched asymptotic expansions for bent and twisted rods: applications for cable and pipeline laying," Journal of Engineering Mathematics, vol. 38, no. 1, pp. 13-31, 2000.

[11] V. Denoël and E. Detournay, "Multiple scales solution for a beam with a small bending stiffness," Journal of Engineering Mechanics, vol. 136, no. 1, Article ID 006001QEM, pp. 69-77, 2010.

[12] V. Denoël and T. Canor, "Patching asymptotics solution of a cable with a small bending stiffness," Journal of Structural Engineering, vol. 139, no. 2, pp. 180-187, 2013.

[13] A. B. Mehrabi and H. Tabatabai, "Unified finite difference formulation for free vibration of cables," Journal of Structural Engineering, vol. 124, no. 11, pp. 1313-1322, 1998. 


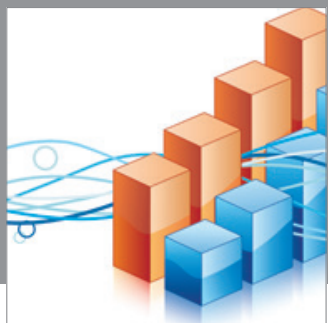

Advances in

Operations Research

mansans

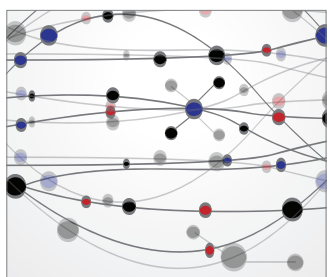

The Scientific World Journal
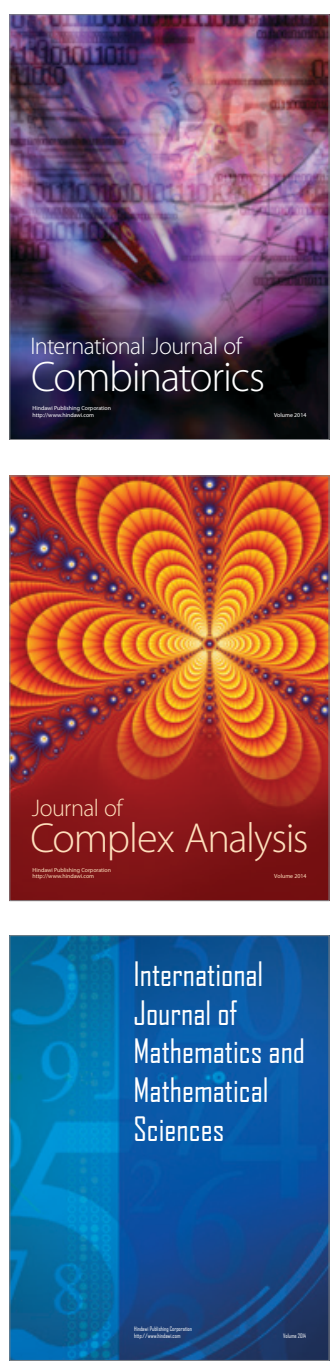
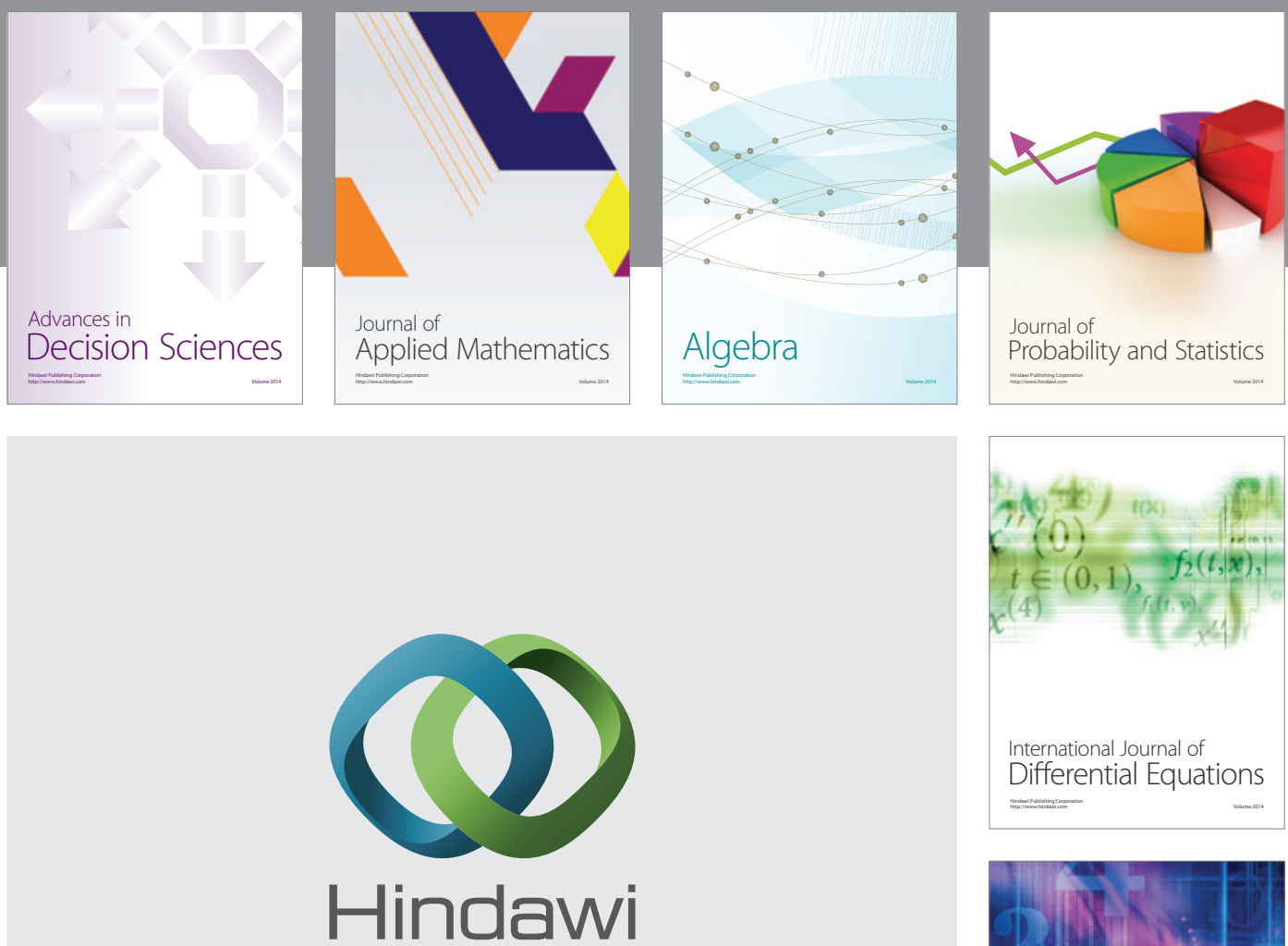

Submit your manuscripts at http://www.hindawi.com
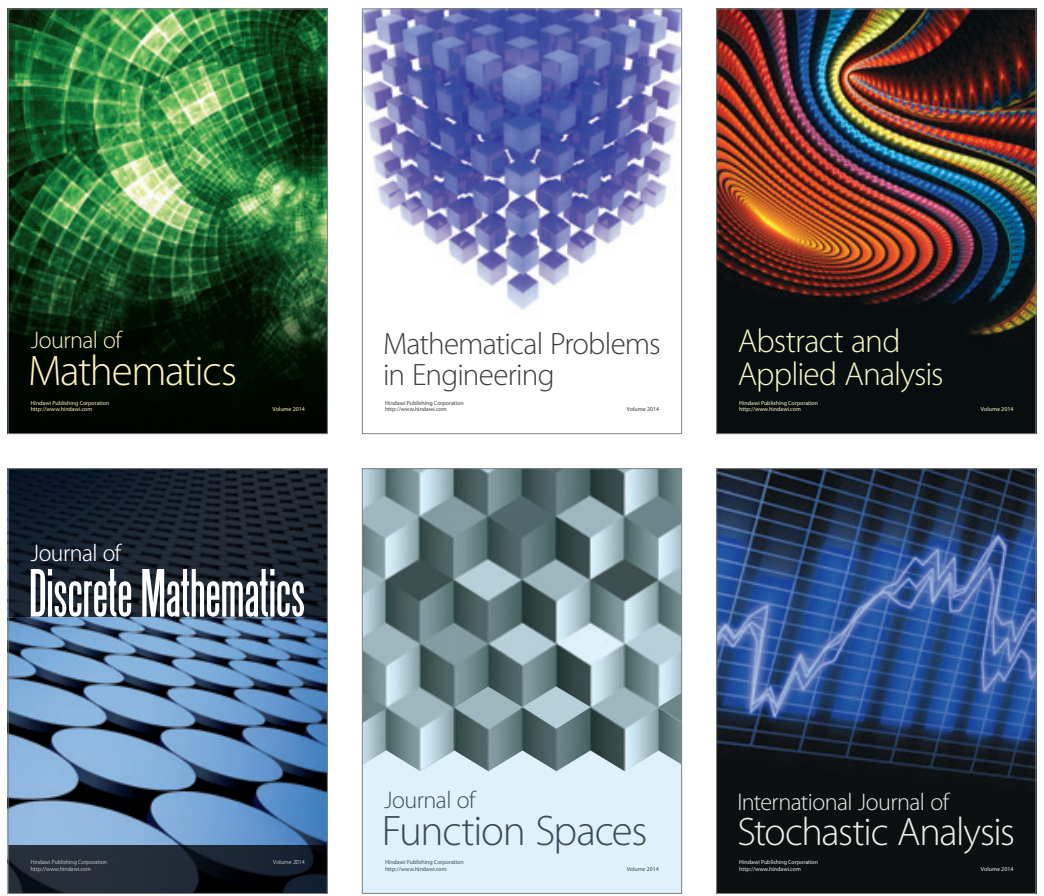

Journal of

Function Spaces

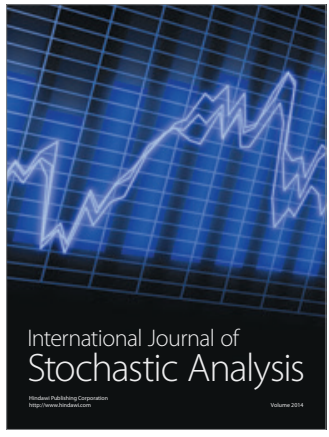

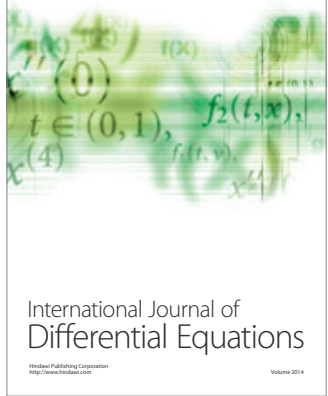
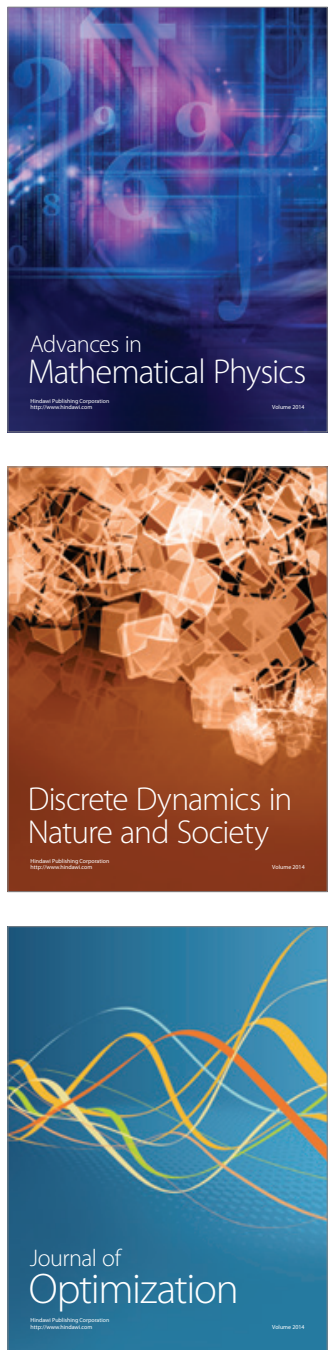